\title{
Photospheric flows measured with TRACE II. Network formation
}

\author{
J. M. Krijger ${ }^{1}$ and T. Roudier ${ }^{2}$ \\ 1 Sterrekundig Instituut, Postbus 80 000, 3508 TA Utrecht, The Netherlands \\ ${ }^{2}$ Laboratoire d'Astrophysique de Toulouse, Observatoire Midi-Pyrénées, 14 Avenue E. Belin, 31400 Toulouse, France \\ e-mail: roudier@bagn.obs-mip.fr
}

Received 26 July 2002 / Accepted 7 March 2003

\begin{abstract}
We analyse a $7 \mathrm{~d}(167 \mathrm{~h})$ sequence of TRACE white-light images with $1^{\prime \prime}$ angular resolution taken at 1 min cadence. The TRACE resolution and the fast cadence allows us to produce maps of the horizontal flow fields with high angular $\left(1^{\prime \prime}\right)$ and temporal resolution $(5 \mathrm{~min})$. The field of view of $128^{\prime \prime} \times 128^{\prime \prime}(\approx 93 \mathrm{Mm} \times 93 \mathrm{Mm})$ covers approximately an area of 10 to 30 supergranules. This area was followed during solar rotation. Magnetic flux was artificially inserted into the successive flow maps in the form of ephemeral regions with positive and negative polarity. The emergence rate of $2 \times 10^{22} \mathrm{Mx} \mathrm{h}^{-1}$ with an average flux per region of about $1.1 \times 10^{19} \mathrm{Mx}$ produces a good reproduction of the chromospheric network as observed in images taken simultaneously at $1600 \AA$. In addition, we show that the quiet network can be maintained only if field elements of both polarities are inserted into the flow fields. Our analysis suggests that the network is fully replenished on a time scale of a day and the lifetimes of the magnetic elements are of a similar duration.
\end{abstract}

Key words. Sun: photosphere - Sun: chromosphere - Sun: granulation

\section{Introduction}

This paper addresses the formation process of the quiet-sun chromospheric network by inserting artificial magnetic elements in observed velocity fields employing long-duration white-light image sequences obtained with Transition Region and Coronal Explorer (TRACE) (Handy et al. 1999).

The chromospheric network consists of chains of bright patches visible in ultraviolet (UV) or Ca II observations. Each patch corresponds to a concentration of magnetic elements in the photosphere with fluxes in the range of $10^{18} \mathrm{Mx}$ up to a few times $10^{19} \mathrm{Mx}$ and field strengths in the order of a kilogauss, commonly modelled as flux tubes (e.g., Steiner et al. 1986). These photospheric elements appear as the bright grains visible in high-resolution Fraunhofer $G$-band (around $\lambda=4305 \AA$ ) images or movies (e.g., Muller et al. 1989; Title \& Berger 1996; Berger et al. 1998; movies on the Dutch Open Telescope website at URL http://dot . astro.uu.nl). On snapshot images the chromospheric network can hardly be separated from the intermittent internetwork (corresponding to the supergranular cell interior) brightenings. However, when averaged over long duration the network stands out since the transient internetwork features are averaged out.

Ever since the first discovery of supergranules (Stuart \& Rush 1954) and supergranular flow (Leighton et al. 1962), their effect on magnetic flux and the network formation has been

Send offprint requests to: J. M. Krijger,

e-mail: J.M.Krijger@astro.uu.nl studied (e.g., Parker 1963; Simon \& Leighton 1964; Rieutord et al. 2000). Magnetic flux concentrations emerge intermittently in the internetwork in the form of ephemeral regions, i.e., bipoles with an average magnetic flux in the order of $10^{19} \mathrm{Mx}$ (Harvey-Angle 1993; Hagenaar 2001).

After their initial emergence the bipoles quickly drift apart and fragment into individual magnetic elements which apparently float passively on the (super)granular flow. The associated flux merges with the existing network or cancels when it encounters opposite-polarity network elements (Schrijver et al. 1997).

Simon et al. (2001, henceforth STW01) constructed a twodimensional model describing horizontal supergranular flows in which they inserted artificial test particles continually in order to simulate the emergence of ephemeral regions. With this procedure they were able to steadily sustain a model representation of the magnetic network. So far, no observational sequence of sufficient duration existed to measure the actual horizontal flow fields over the supergranular lifetime of a few days, which would enable a check of their model.

The SOHO and TRACE missions provide continuous solar observing capability with sufficient cadence, angular resolution and pointing stability to permit such long-duration flow mapping. A study of this type by Lisle et al. (2000, henceforth LRT00) employed a 6 d observation with the Michelson Doppler Interferometer (MDI) aboard SOHO, however in lowresolution mode with only 4 " resolution. Details of the MDI instrument are given by Scherrer et al. (1995). 
Another study of this type employing MDI was done by Shine et al. (2000) using the high-resolution mode. Although MDI then offers $1.3^{\prime \prime}$ resolution $\left(0.65^{\prime \prime}\right.$ pixels in highresolution mode) at the required cadence, the duration of the observation was limited (as are all MDI observations in this mode) by the fixed pointing of the field with a size of about $600^{\prime \prime} \times 600^{\prime \prime}$. Solar rotation limits the maximum duration of a continuous observation to about two days for a solar area about 300" wide. TRACE has a somewhat better resolution $\left(1^{\prime \prime}\right)$ and its pointing capability permits tracking a fixed solar disk area while it rotates around half the sun. The foreshortening of such an area near the limb limits the effective duration of such sequences to between $5 \mathrm{~d}$ to $7 \mathrm{~d}$. As shown in the first paper of this series (Krijger et al. 2002, henceforth Paper I), TRACE white-light images can be used to study horizontal velocity fields at a 5 min temporal and $1^{\prime \prime}$ angular resolution.

The organization of the paper is as follows. The observations are presented in the next section. In Sect. 3 we briefly describe our local correlation tracking method. The cork insertion techniques are described in Sect. 4. In Sect. 5 the results of the analysis are presented with the discussion and conclusion following in Sects. 6 and 7, respectivily.

\section{Observations}

Between 00:44 UT April 22, 2000 and 00:09 UT April 29, 2000, TRACE tracked a region mostly free of active regions across the solar disk along the equator between Stonyhurst latitude $45 \mathrm{E}$ and $45 \mathrm{~W}$ for $167.25 \mathrm{~h}$. The satellite pointing was changed several times to correct for the solar rotation and keep the same region in the used field of view of $768 \mathrm{px} \times 768 \mathrm{px}$ (or $384^{\prime \prime} \times 384^{\prime \prime}$, since the TRACE pixel size is $0.5^{\prime \prime}$ ). Only part of the CCD was used to increase the cadence of the whitelight continuum images to at least 1 min (EUV $171 \AA$ images were taken as well, but are not used in this study). Some gaps appear in the data due to planned synoptic observations or errors in the data transfer. There are only nine gaps longer than $10 \mathrm{~min}$, with the longest lasting $45 \mathrm{~min}$. Since supergranular flows evolve on time scales of hours to days, these gaps do not impair the analysis presented in this paper. When sufficient bandwidth was available (about half the time) also $1600 \AA$ passband images of the same field were taken at a $1 \mathrm{~min}$ cadence. See Fig. 1 for samples of white-light and $1600 \AA$ images. The white-light image shows the granulation with a small pore in the upper central part. The $1600 \AA$ image shows the same field with the internetwork appearing dark with intermittent bright grains. The network is present as bright patches, corresponding to the non-visible magnetic network in the underlying photosphere.

The co-alignment of the image was done by R.A. Shine and is described in more detail in Shine et al. (2001).

All white-light images were carefully co-aligned using cross-correlation. The useful field of view shrank to $287^{\prime \prime} \times$ $320^{\prime \prime}$ due to the effect of solar rotation and satellite drift. After a temporal gap the co-alignments retain an amount of uncertainty because solar patterns evolved during the temporal gap. The images of the other TRACE passbands were aligned to the white-light by adjusting for the offset in pointing between

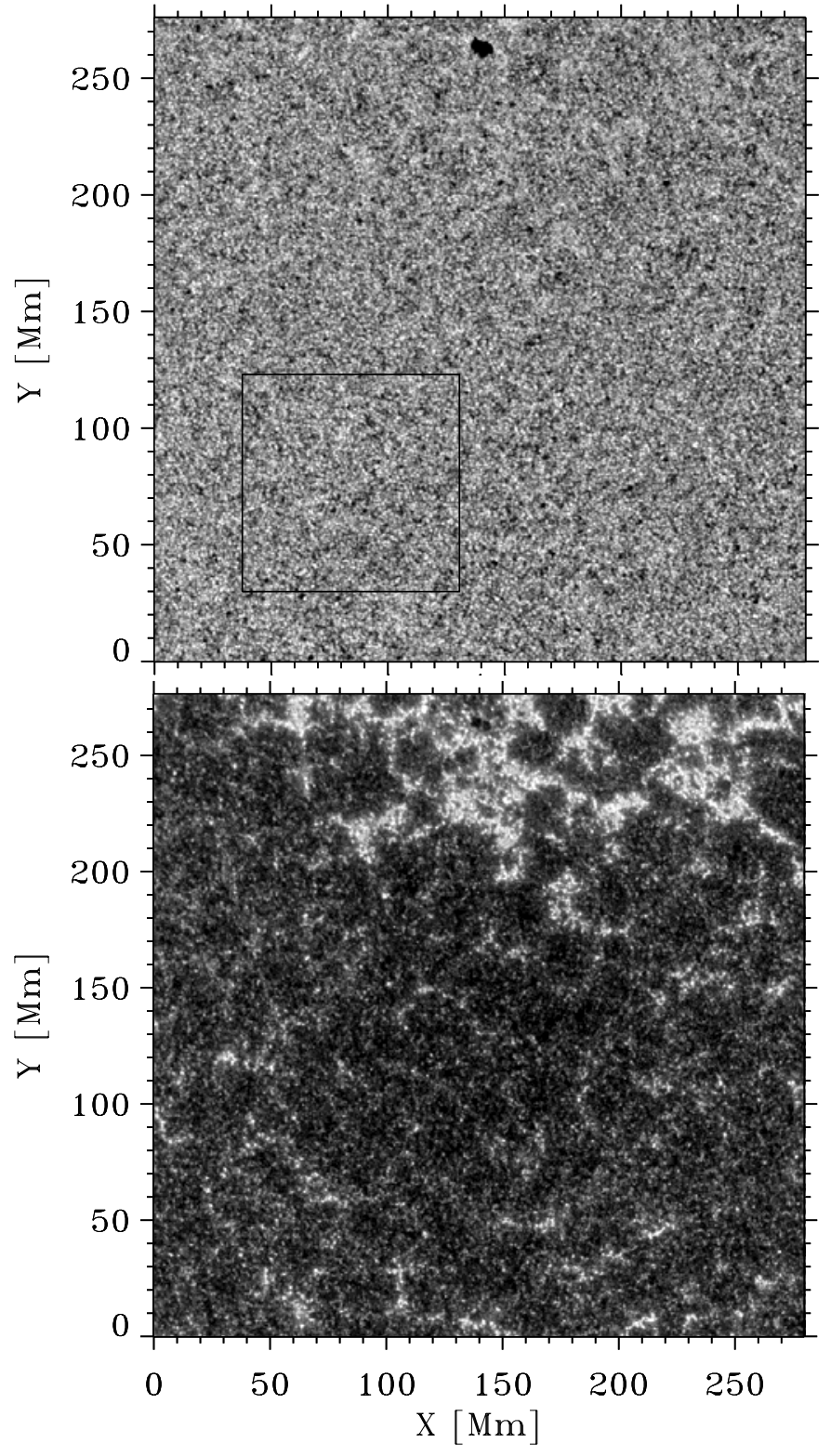

Fig. 1. Top: sample TRACE white-light image obtained at April 25, 2000, 13:55 UT. Bottom: sample $1600 \AA$ passband image obtained $58 \mathrm{~s}$ earlier. The solid box in the upper panel indicates the subfield for which we derived the velocity field.

different passbands and by interpolation from the derived white-light displacements.

\section{Local correlation tracking}

Due to computer memory limitation we only used a $256 \mathrm{px} \times$ 256 px subfield corresponding to $128^{\prime \prime} \times 128^{\prime \prime}$ or about $93 \mathrm{Mm} \times 93 \mathrm{Mm}$, indicated by the square contours in the upper panel of Fig. 1.

We use the technique of Roudier et al. (1999) and Paper I and briefly describe it here. Each individual white-light image was four times enlarged through interpolation. This data resampling makes the subsequent correlation computations more precise because the noise is proportional to the square root of the number of samples used in the calculations. Each resampled 
subfield was then spatially filtered using a $1^{\prime \prime}$ Gaussian window. Subsequently the local curvature of the intensity pattern was used to derive a binarized granule map following Strous (1995).

From each pair of successive images we measured the horizontal flow field by dividing the first image into $1^{\prime \prime} \times 1^{\prime \prime}(8 \mathrm{px} \times$ $8 \mathrm{px})$ patches and determining the vector translation of the patch (as marked by its fine structure) to its position in the next image through local cross-correlation. Using this Local Correlation Tracking (LCT) method of November \& Simon (1988) on binarized maps was named $\mathrm{LCT}_{\text {bin }}$ by Roudier et al. (1999). To reduce noise we average these offsets over $5 \mathrm{~min}$, a good compromise between granule lifetime and the resulting flow map noise. Any shift found outside the $3 \sigma$ limit due to cross-correlation failure was replaced by the average offset of the surrounding eight patches. Conversion of the pixel translation vectors then resulted in a sequence of flow field maps with a 5 min temporal and $1^{\prime \prime}$ angular resolution. Missing maps due to data gaps were interpolated from the preceding and following velocity images. Various systematic uncertainties related to the distance to the solar limb were corrected for by subtracting the average over each flow map, as suggested by Shine et al. (2000).

A useful property that can be derived from horizontal flow maps is the divergence. The divergence at location $(i, j)$ in a regular discrete grid is defined as:

$\frac{v_{i}(i+1, j)-v_{i}(i-1, j)+v_{j}(i, j+1)-v_{j}(i, j-1)}{2 W}$,

with $W$ the distance between two grid points and $v_{i}, v_{j}$ the respective velocity components. Divergence maps show where matter presumably disappears from the surface by flowing downwards (convergence) and where matter presumably appears at the surface by flowing upward (divergence). Figure 2 shows a sample flow and divergence map, respectively. Diverging and converging patterns at mesoscale $(\approx 7 \mathrm{Mm})$ dominate the $5 \mathrm{~min}$ average flow map. The distribution of the flow speed magnitudes has a maximum around $0.6 \mathrm{~km} \mathrm{~s}^{-1}$ (Fig. $3^{1}$ ). It is nearly identical to the distribution found by Shine et al. (2000). The lower limit detectability is determined by the (resampled) TRACE CCD pixel size, the cadence, and the subpixel accuracy of the LCT method.

\section{Cork insertion}

Corks are hypothetical particles that are allowed to flow freely in the horizontal flow maps (Simon et al. 1988). At each time step the position of the cork is updated according to the velocities on the location of the cork at that time step.

The pioneering flow study of Simon et al. (1988) used data of such short duration $(20 \mathrm{~min}$ ) that the velocity field had to be extrapolated to a much longer duration. Later studies, e.g., Brandt et al. (1992) and Shine et al. (2000) used data durations of several hours, still much shorter than the multi-day lifetime

\footnotetext{
${ }^{1}$ Note that the amplitude of the flow speed derived in similar fashion in Paper I was much smaller because an error was made in the numerical interpolation.
}

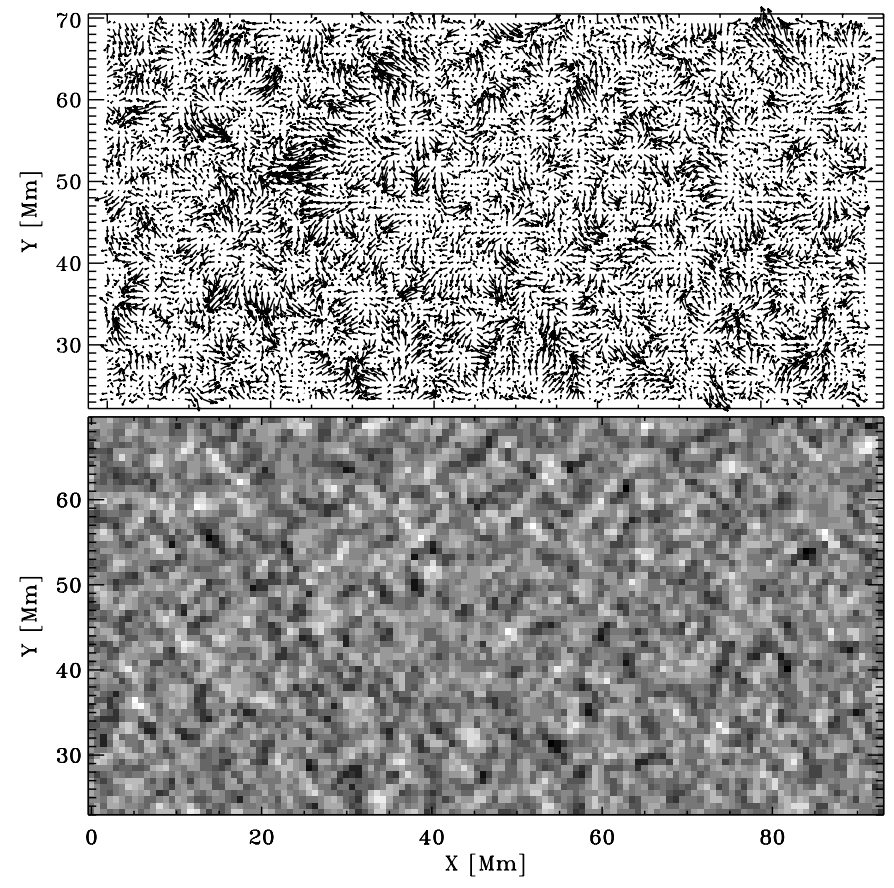

Fig. 2. Top: sample flow map. Shown is the central half of the horizontal velocity field between 13:51 and 13:56 UT, 25 April 2000. Bottom: divergence map of the same horizontal velocity field. Converging areas where matter presumably disappears from the surface by flowing downwards are dark in greyscale, while diverging areas where matter presumably appears at the surface by flowing upward are bright. Axes: distance from the lower-left origin of the observational field of view in units of megametre.

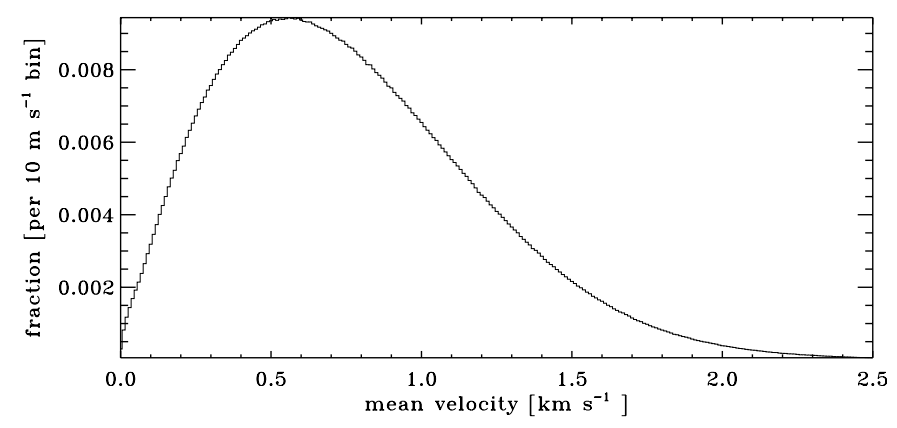

Fig. 3. Distribution of the horizontal flow in the flow map sequence. The standard deviation is about equal to the thickness of the curve.

of supergranules. The long sequence used for this study shows that the network and flow field evolve significantly over several days. The most common method of using corks is to insert all corks initially at regular spacing across the entire velocity field, and then follow them as they flow. The results of this technique applied to our data is shown in Fig. 4. Each concentration of corks is indicated by a circle with an area propertional to the number of overlapping corks on the central location. After $90 \mathrm{~h}$ only a few very dense corks concentrations can be distinguished because the corks end up in strong convergence points ("sinks") or are moved outside our observed field. To study the magnetic network over a period as long as our observation, a more sophisticated cork insertion method is needed including cork cancellation due to opposite polarity. 


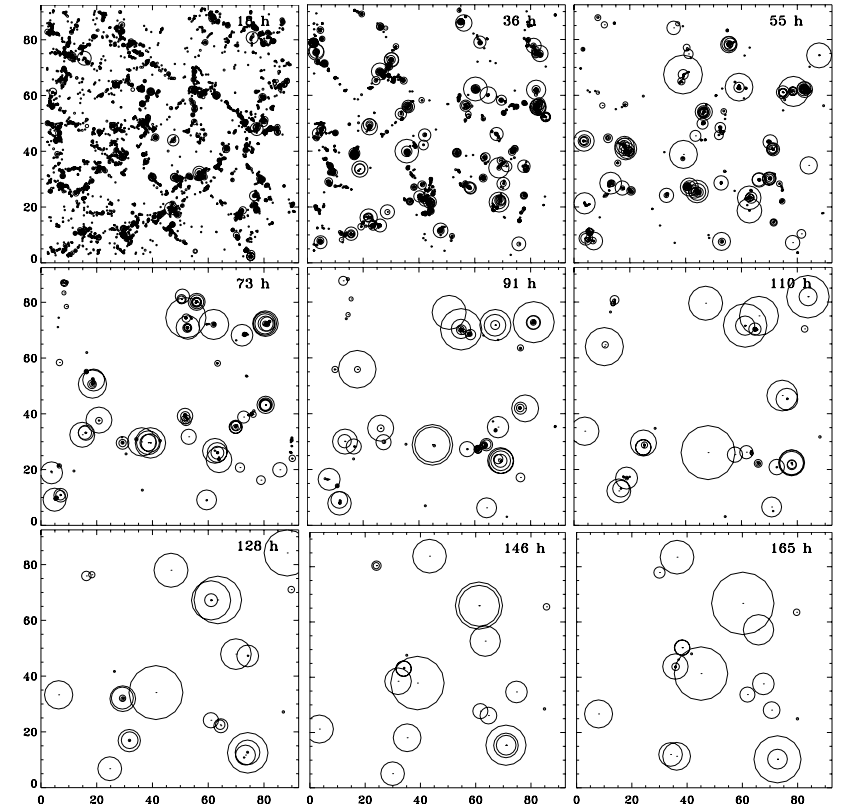

Fig. 4. Evolution of an initially regular distribution of $128 \times 128$ corks, each with a size $0.1^{\prime \prime} \times 0.1^{\prime \prime}$. Overlapping corks are replaced by a circle with an area proportional to the overlapping number of corks. Time elapsed since the insertion of the corks is indicated in the upper right-hand corner of each panel. Axes are units of megametre.

We follow STW01 in comparing our cork insertion technique to the ephemeral active regions observed with MDI (Schrijver et al. 1997). Most of the solar magnetic flux emerges in the form of small bipoles, called ephemeral regions (Harvey-Angle 1993; Hagenaar 2001). After their initial emergence the bipoles split and quickly drift apart apparently floating passively on the (super)granular flow. According to this method, new corks are introduced as bipole structures similar to ephemeral regions consisting of two opposite-polarity clusters of corks. For comparison purposes we initially use the same values for the different parameters that are used in STW01. A cork possesses a position and a sign (either positive of negative). As we do not know the position of the supergranules we inserted the centre of each bipole structure at a random position anywhere in the inner two thirds of the measured velocity field. The clusters are always separated by the same distance $D\left(10^{\prime \prime}\right)$ and contain the same number of unipolar corks (20). The corks in each cluster are distributed in a Gaussian pattern with a standard deviation of 1" around the centre of the cluster. Figure 5 shows a typical sample of such a bipolar structure. After inserting the bipole structure we allow the corks to flow with the flow map speeds, sampling the flow maps at their 5 min intervals. If two corks of opposite sign come within $0.1^{\prime \prime}$ they cancel each other. Corks that are carried off the edges of the field are accumulated until enough corks of either sign are available to form a new bipole structure which is then inserted in the central two-thirds area of the field again. This minimizes the loss of corks at the field edges.

According to Schrijver et al. (1997) magnetic flux concentrations emerge intermittently at a rate of $3 \times 10^{21} \mathrm{Mx} \mathrm{h}^{-1}$ over the whole solar surface. The average magnetic flux of

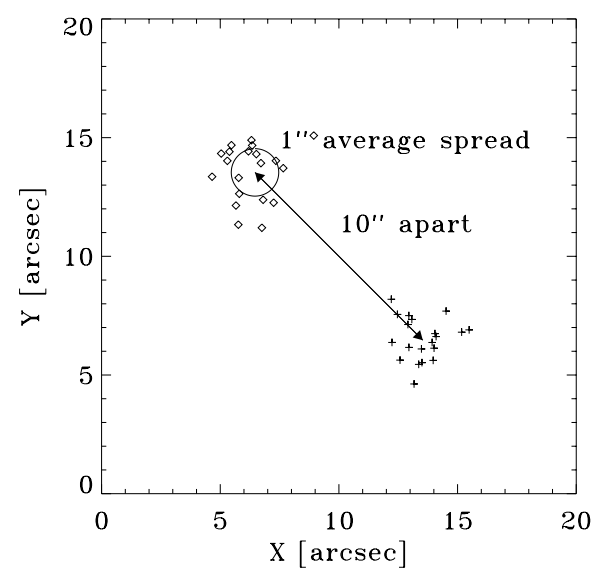

Fig. 5. An sample of the type of bipolar cork structures inserted into the velocity field. The centres of the two clusters are always $10^{\prime \prime}$ apart. Each cluster consists of 20 corks of the same sign, while the corks themselves are distributed in a random pattern with Gaussian cork density distribution with a standard deviation of $1^{\prime \prime}$ around the cluster centre location. Locations of corks with a positive sign are indicated with a plus, corks with negative sign with a diamond.

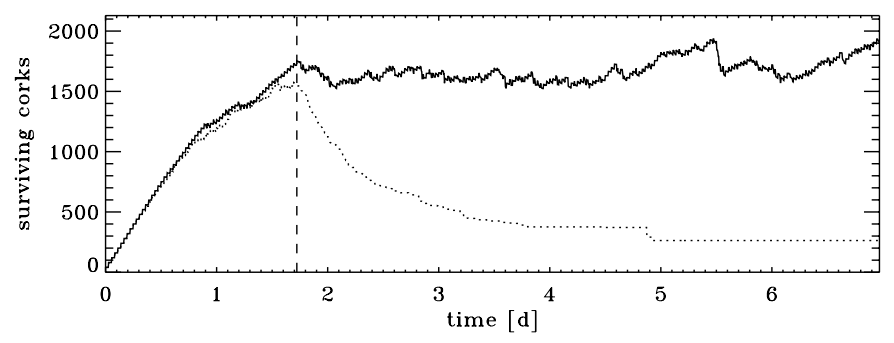

Fig. 6. Solid curve: number of surviving corks as a function of time with new clusters of corks inserted every $40 \mathrm{~min}$. Dotted curve: similar cork survival when the insertion is halted after $1.7 \mathrm{~d}$ (indicated by the vertical dashed line).

ephemeral regions is in the order of $10^{19} \mathrm{Mx}$ (Harvey-Angle 1993; Hagenaar 2001).

In STW01 where each cork represents a fluxtube with a $1500 \mathrm{G}$ field strength and a magnetic flux of $7.5 \times 10^{16} \mathrm{Mx}$, the resulting flux for each bipole structure would be $3 \times$ $10^{18} \mathrm{Mx}$, which implies that on average 1000 ephemeral regions appear each hour on the sun. To simulate this we insert a new bipolar structure every $40 \mathrm{~min}$ in our $128^{\prime \prime} \times 128^{\prime \prime}$ field.

Another possible source of magnetic flux consists of elusive weak internetwork magnetic patches or elements (Wang et al. 1995; Lin 1995; Zhang et al. 1998; Lin \& Rimmele 1999). These magnetic elements play only a minor role in sustaining the magnetic network (Martin 1990; Wang et al. 1996; Zhang et al. 1998) and have been ignored.

\section{Results}

We use the cluster-insertion recipe presented in Sect. 4 to sprinkle bipolar cork groups onto the sequence of flow maps derived from the TRACE images described in Sect. 2. Figure 6 shows results concerning the total cork number evolution. The dotted curve shows the surviving cork number when the insertion of new clusters (every $40 \mathrm{~min}$ ) is stopped after a total of 
2480 corks have been inserted after $1.7 \mathrm{~d}$. The sudden drops mark occasions at which whole unipolar clusters meet and cancel clusters of the opposite sign.

The solid curve in Fig. 6 shows the surviving cork number when new clusters are inserted every $40 \mathrm{~min}$ during the whole duration of the experiment. After the initial build-up of the cork distribution which takes about two days, a statistically steady state develops with $N \approx 1600$. An insertion rate of 40 corks every 40 min with $N \approx 1600$ means that the network is fully refreshed every $1600 \mathrm{~min}$ or within almost $27 \mathrm{~h}$, half the value found by SWT01. The average lifetime of the corks must have the same order of magnitude. The modelled supergranular velocity field of STW01 resembles our and other observations, so only their granular displacement and assumed supergranule radii can make a difference. The granular displacement of STW01 that created a network most similar to other observations was $0.5^{\prime \prime}$ per $10 \mathrm{~min}$, which equals an average speed of $0.6 \mathrm{~km} \mathrm{~s}^{-1}$, the same mean speed as found by us. As STW01 showed, the lifetime of corks decreases when supergranules are smaller as the corks travel smaller distances to the supergranule borders where they can be cancelled. Our findings support such a smaller supergranule radius, in agreement with the supergranule sizes found by Hagenaar et al. (1997) of the order of $13 \mathrm{Mm}$ to $18 \mathrm{Mm}$. Figure 7 shows the evolution of the network for this continuous cluster insertion similar to Fig. 4. The corks outline supergranular boundaries as the pattern evolves over time.

Figure 8 shows on the left-hand side two temporal averages of TRACE $1600 \AA$ image sequences with durations of $50 \mathrm{~min}$. Their mid-times are indicated in each panel. The contours that are superimposed on the average images show the smoothed zero-divergence levels. On the right hand side the cork positions halfway the averaging interval are indicated by plus symbols for positive-polarity corks, diamonds for negative-polarity corks. The zero-divergence contours are repeated for ease of comparison. The network coincides with the converging areas as shown in Paper I. There is also generally a spatial coincidence between the cork positions and the chromospheric network. However, it seems that the cork density is too low to reproduce the chromospheric network fully. The network in the outer part of the field is not well reproduced as the corks were all inserted in the inner two thirds of the field.

Figure 9 displays various cork properties. The first panel displays the lifetime $\tau$ distribution for the corks. Corks that have not yet cancelled or are accumulated at the edges of the field at the end of our flow map sequence are ignored. This has little effect since these corks form only a minor fraction of the total amount of corks. While the cork is moved around by the instantaneous flow patterns, it travels over a total path length $d_{\mathrm{a}}$ between its insertion and its eventual cancellation. The second panel in Fig. 9 displays the total path length $d_{\mathrm{a}}$ distribution. From these two quantities we can derive the average actual velocity $\left\langle v_{\mathrm{a}}\right\rangle$ shown in the third panel. Not surprisingly, the histogram of $\left\langle v_{\mathrm{a}}\right\rangle$ displayed in the fourth panel, shows as a sharply peaked distribution around the original mean solar flow velocity in Fig. 3. Yet corks are following a flow, which at small time scales resembles a random walk so the net displacement $d_{\mathrm{n}}$ is much smaller than the total path length $d_{\mathrm{a}}$. The

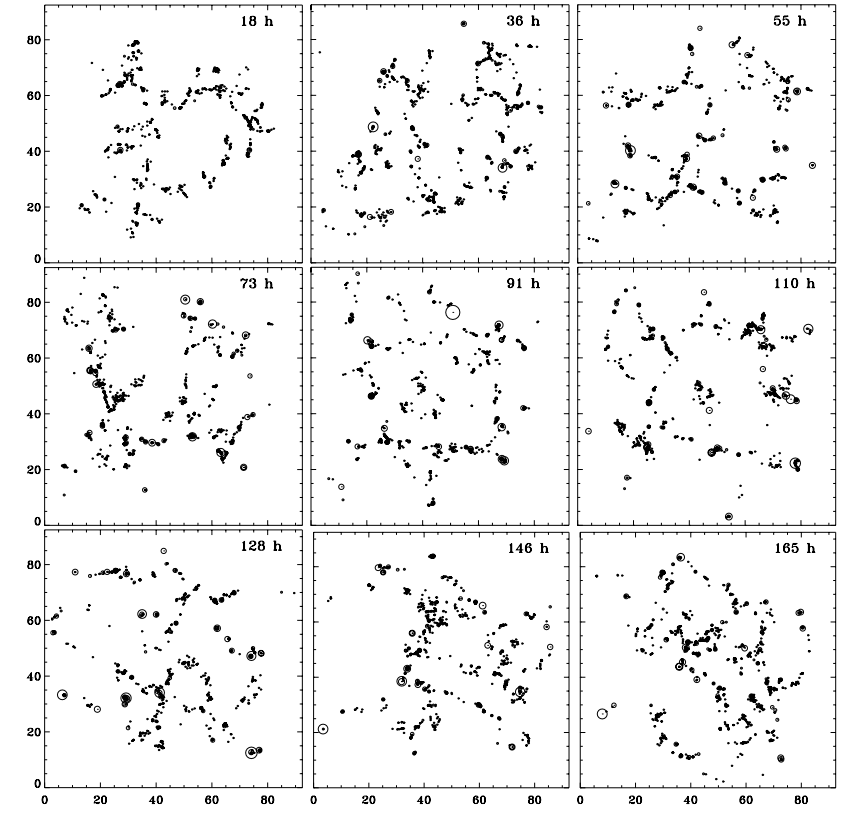

Fig. 7. Formation of a statistically steady network, same format as Fig. 4. Corks were inserted into the observed flow maps in bipolar clusters as illustrated in Fig. 5 every 40 min. Axis units in megametre.

fourth panel of Fig. 9 displays the net displacement $d_{\mathrm{n}}$ distribution. The distribution of the net-speed $v_{\mathrm{n}}$, derived from the lifetime $\tau$ and the net displacement $d_{\mathrm{n}}$, is displayed in the fifth panel. The net displacements $d_{\mathrm{n}}$ are ten times smaller than the actual displacement $d_{\mathrm{a}}$. This difference illustrates that the corks are moving in random-walk fashion due to the buffeting by individual granules.

Figure 10 shows the correlation between the lifetime $\tau$ and the actual speed $\left\langle v_{\mathrm{a}}\right\rangle$ and the correlation between lifetime $\tau$ and the net speed $v_{\mathrm{n}}$, respectively. The former displays a sharply peaked profile around the mean value of the measured flows (Fig. 3). This can be explained with each displacement between time steps being considered as a statistical draw from the parent velocity distribution (multiplied by the time step size). Corks with a long lifetime had more draws from this distribution and will most likely have an actual speed $\left\langle v_{\mathrm{a}}\right\rangle$ close to the mean of the parent distribution. We find a weak inverse correlation between the net velocity $v_{\mathrm{n}}$ and lifetime $\tau$.

Our corks live considerably longer than the magnetic elements of LRT00 or Hagenaar et al. (1999) as can be seen in Fig. 9. As our corks live until they are cancelled it is clear that besides cancellation an additional process must be responsible for shortening the lifetime of real magnetic elements.

\subsection{Increased emergence rate}

So far we have used the same emergence rate as STW01, namely $3 \times 10^{21} \mathrm{Mx} \mathrm{h}^{-1}$ over the whole solar surface. This corresponds to one ephemeral region of $3 \times 10^{18} \mathrm{Mx}$ per $40 \mathrm{~min}$ in our $128^{\prime \prime} \times 128^{\prime \prime}$ field. Using a $3.5 \mathrm{~d}$ MDI observation Hagenaar (2001) found a much higher value for the flux emergence rate, namely $2 \times 10^{22} \mathrm{Mx} \mathrm{h}^{-1}$, with an average flux per region of about $1.1 \times 10^{19} \mathrm{Mx}$. To match these observations 

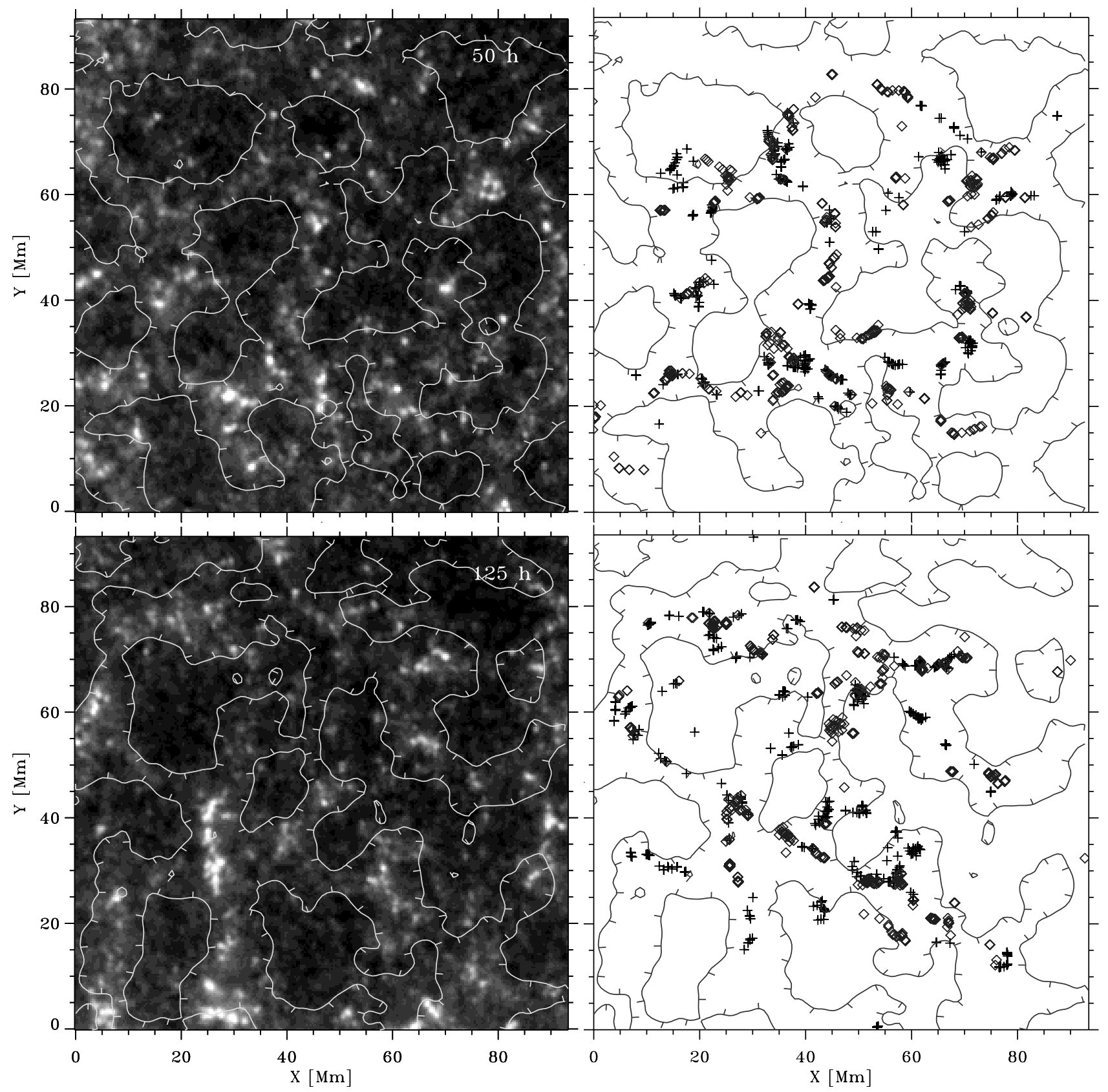

Fig. 8. Greyscale images: sample temporal averages over 50 min duration TRACE $1600 \AA$ images. The greyscale is logarithmic in order to bridge the contrast between network and internetwork. The contour curves mark the transition between divergence and convergence, with ticks directed to converging regions. The mid-times of the averaging periods are specified in the upper right-hand corner in minutes after the start of the observing run. Scatter plots: locations of the corks at mid-time of the averaging period; positive signs are indicated by a plus, negative signs by a diamond. The zero-divergence contours are repeated.

we inserted a bipole structure every $25 \mathrm{~min}$, each consisting of a total of 150 corks. This results only in an increase by a factor of 6 , instead of $\approx 6.7$, because we are limited by our 5 min time-step resolution. The total number of corks in the steady-state network in our field increases from $N \approx 1600$ to $N \approx 7000$. As expected, $N$ does not increase proportionally to the six times larger insertion rate because the cancellation rate increases also. The lifetime $\tau$ of the corks decreases significantly. The lifetime distribution peak shifts to $14 \mathrm{~h}$, the same period needed to replace all the magnetic field in the network found by Hagenaar (2001). Figure 11 displays a superposition similar to Fig. 8, but using the larger cork insertion rate. The actual chromospheric network is much better outlined by the corks here than in Fig. 8. Again the network in the outer part of the field is not well reproduced as the corks were all inserted in the inner two thirds of the field.

\section{Discussion}

Use of the emergence rate of $3 \times 10^{21} \mathrm{Mx} \mathrm{h}^{-1}$ and $3 \times$ $10^{18} \mathrm{Mx}$ per ephemeral region, i.e., the values found by Schrijver et al. (1997), reproduces the network only sparsely. 

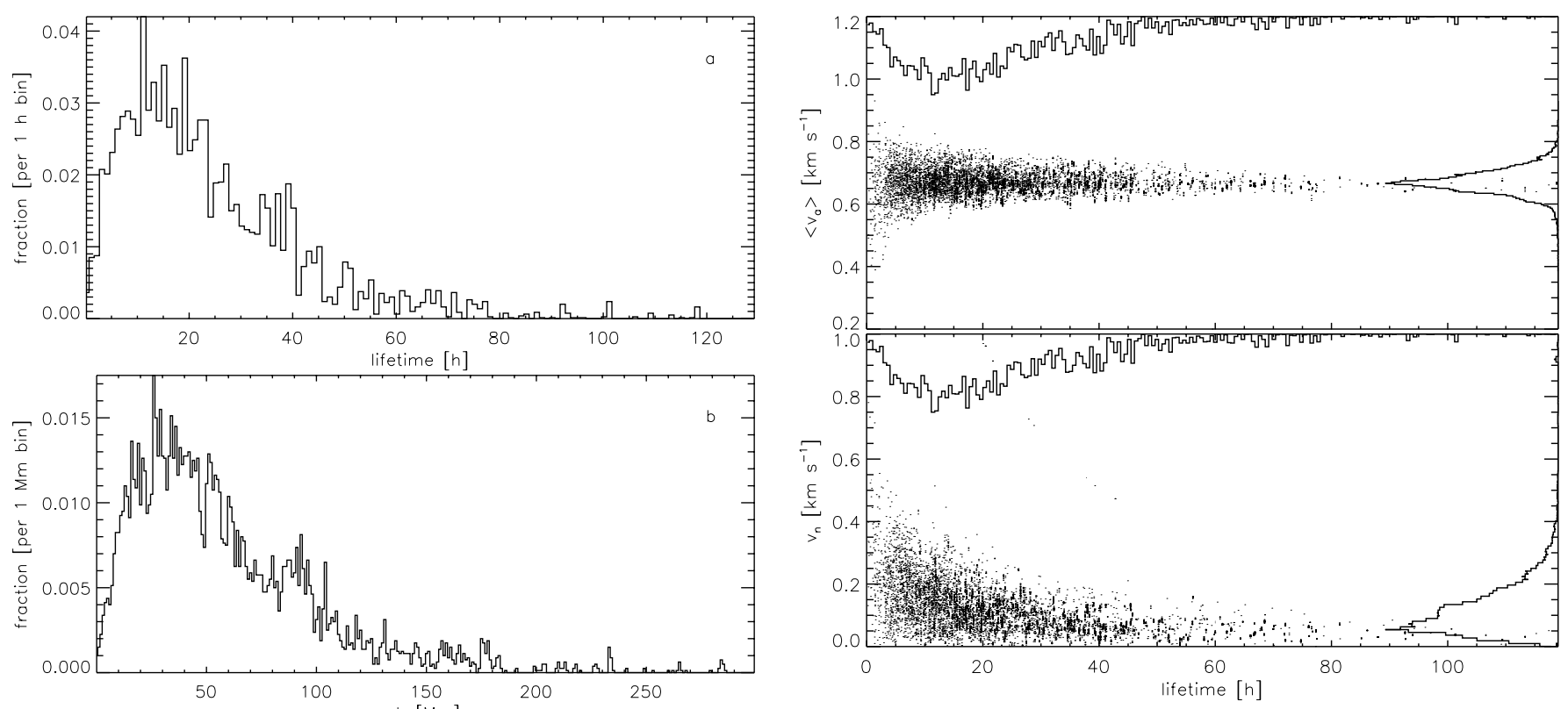

Fig. 10. Scatter plot of the actual speed $\left\langle v_{\mathrm{a}}\right\rangle$ (top) and the net velocity $v_{\mathrm{n}}$ (bottom) against the life times $\tau$, respectively. The solid curves show the inverted, normalized distributions.

Hagenaar (2001) produces a much better outline of the actual network.

There are some differences between our study and the work of STW01. In STW01 the bipole cork structure was inserted within $5^{\prime \prime}$ of the centre of a randomly selected supergranule. In our method we do not know the position of the supergranules before inserting the corks into the velocity field. We have thus inserted the centre of the bipole structure at a random position anywhere in the inner two thirds of the measured velocity field. This insertion method can result in some corks being inserted closer to the supergranule boundaries and thus having to travel less far before reaching the network where there is a larger chance of being cancelled. Also, indications exist that ephemeral regions preferably appear close to the boundaries instead of the centre of supergranules (Harvey \& Harvey 1995). However, STW01 tested the effect of increasing the area within the supergranule where the ephemeral regions could emerge and found it has little effect on the resulting network.

In STW01 the positions of the corks are updated in a $10 \mathrm{~min}$ time step scheme, while we use a 5 min time step. A test (not presented here) using 10 min average velocity maps showed that this does not have much effect on the cork statistics.

Following STW01, we have investigated the sensitivity of our results to variations of the parameter values. We find that varying the separation between clusters, $D$, from $5^{\prime \prime}$ to $25^{\prime \prime}$ does not have a great effect on the formed network. Changing the spread of the Gaussian pattern around the clusters centre from $1^{\prime \prime}$ to $0.5^{\prime \prime}$ and $2^{\prime \prime}$ also has little effect, although decreasing the spread of the clusters causes more corks to survive as they are less likely to encounter a cork with an opposite sign. Similarly to STW01, we find that the results are quite insensitive to the parameters of the cork insertion (except for the amount of corks per cluster).

The higher emergence rate of $2 \times 10^{22} \mathrm{Mx} \mathrm{h}^{-1}$ with an average flux per ephemeral region of about $1.1 \times 10^{19} \mathrm{Mx}$ found by
Fig. 9. From top to bottom: histograms of a) cork lifetime $\tau$; b) actual cork travel distance measured along the cork path $d_{a}$; c) actual cork speed $\left\langle v_{\mathrm{a}}\right\rangle$ along its path averaged over the lifetime of the cork; d) net cork displacement $d_{\mathrm{n}}$ between insertion and cancellation; e) net cork speed $v_{\mathrm{n}}$. 


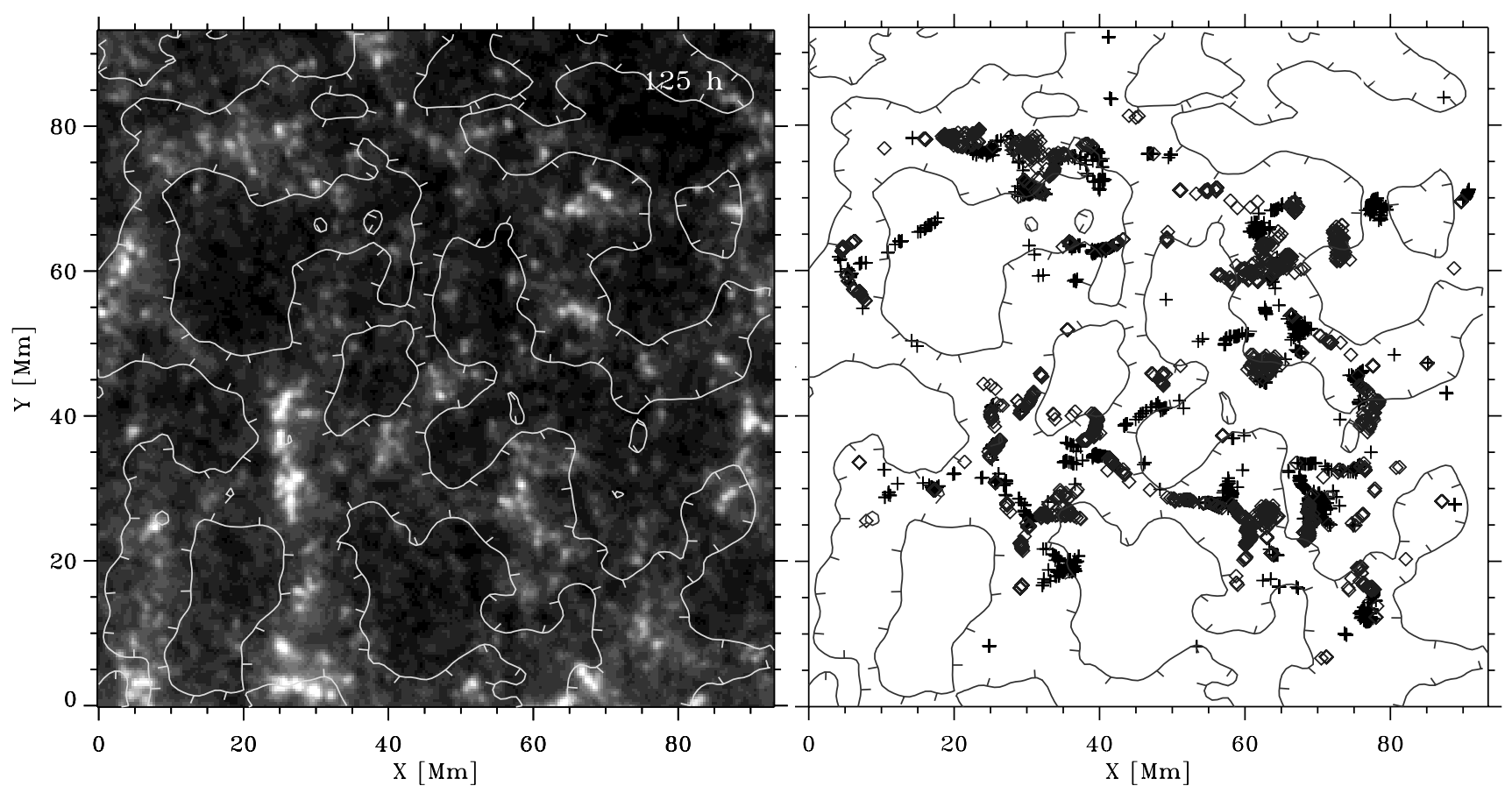

Fig. 11. Similar to Fig. 8 but now with a six times higher flux emergence rate.

There is an inverse correlation between the net velocity $v_{\mathrm{n}}$ and lifetime $\tau$. A similar but stronger correlation was seen by LRT00, but the life times found by LRT00 are significantly shorter than ours. This might be due to correlation between the lifetime and the magnetic field strength of a magnetic element, as shown by LRT00 and Schrijver et al. (1997). Shortlived and weak magnetic elements occur more frequently than strong, long-lived elements. Since our corks all have the same amount of magnetic flux we do not include such short-lived magnetic elements in our modelling. Future modelling of magnetic elements using artificial corks will not only have to take into account the magnetic sign but also the magnetic strength of the corks.

\section{Conclusion}

We have measured solar surface flows at high angular ( $\left(1^{\prime \prime}\right)$ and temporal $(5 \mathrm{~min})$ resolution over a much longer duration than has been achieved so far, sufficiently long to permit flow measurements over time scales covering the full range from granular to supergranular evolution. Confirming the work of STW01, we have shown the necessity to include cork polarity. We inserted magnetic flux into the supergranules in the form of ephemeral regions consisting of a number of corks with either positive or negative polarity. In order to maintain the network longer than two days continuous insertion of new magnetic elements is needed as older magnetic elements cancel when they encounter opposite polarity flux.

The network is fully replenished on a time scale of a day. The magnetic elements move in a random walk with a preferential direction towards supergranular boundaries as an effect of being buffeted by individual granules. Also the lifetimes for our corks are of the order of a day, confirming the work of STW01 and Schrijver et al. (1997). These lifetimes are in agreement with an average supergranular size of the order of $13 \mathrm{Mm}$ to $18 \mathrm{Mm}$ in the work of STW01. We do not take into account the correlation between the magnetic strength and the lifetime of a magnetic element as found by, e.g., Hagenaar et al. (1999).

Our work shows that the chromospheric network is well reproduced by emerging magnetic elements that passively flow in observed velocity fields when using the higher emergence rate of $2 \times 10^{22} \mathrm{Mx} \mathrm{h}^{-1}$ with an average flux per ephemeral region of about $1.1 \times 10^{19} \mathrm{Mx}$ as found by Hagenaar (2001). However, several improvements can be made to our cork evolution recipe, for example, by introducing weak internetwork fields or a reactive force for each cork related to the intensity of the magnetic field and not consider them a passive scalar, or by removing limitations such as identical magnetic strength and infinite lifetime unless cancelled.

Acknowledgements. J.M. Krijger's research was funded by The Netherlands Organization for Scientific Research (NWO). We also would like to thank R.A. Shine for making the aligned data available to us. We are indebted to R.J. Rutten for many improvements to the manuscript.

\section{References}

Berger, T. E., Löfdahl, M. G., Shine, R. S., \& Title, A. M. 1998, ApJ, 495, 973

Brandt, P. N., Rutten, R. J., Shine, R. A., \& Trujillobueno, J. 1992, in Cool Stars, Stellar Systems, and the Sun, ASP Conf. Ser. 26, 7, 161

Hagenaar, H. J. 2001, ApJ, 555, 448

Hagenaar, H. J., Schrijver, C. J., \& Title, A. M. 1997, ApJ, 481, 988 
Hagenaar, H. J., Schrijver, C. J., Title, A. M., \& Shine, R. A. 1999, ApJ, 511, 932

Handy, B. N., Acton, L. W., Kankelborg, C. C., et al. 1999, Sol. Phys., 187,229

Harvey, K., \& Harvey, J. 1995, Air Force Rep. AFGL-TR-76-0225

Harvey-Angle, K. L. 1993, Magnetic Bipoles on the Sun (Utrecht University: Ph.D. Thesis)

Krijger, J. M., Roudier, T., \& Rieutord, M. 2002, A\&A, 387, 672

Leighton, R. B., Noyes, R. W., \& Simon, G. W. 1962, ApJ, 135, 474

Lin, H. 1995, ApJ, 446, 421

Lin, H., \& Rimmele, T. 1999, ApJ, 514, 448

Lisle, J., De Rosa, M., \& Toomre, J. 2000, Sol. Phys., 197, 21 (LRT00)

Martin, S. F. 1990, IAU Symposium, 138, 129

Muller, R., Hulot, J. C., \& Roudier, T. 1989, Sol. Phys., 119, 229

November, L. J., \& Simon, G. W. 1988, ApJ, 333, 427

Parker, E. N. 1963, ApJ, 138, 552

Rieutord, M., Roudier, T., Malherbe, J. M., \& Rincon, F. 2000, A\&A, 357,1063

Roudier, T., Rieutord, M., Malherbe, J. M., \& Vigneau, J. 1999, A\&A, 349,301

Scherrer, P. H., Bogart, R. S., Bush, R. I., et al. 1995, Sol. Phys., 162, 129
Schrijver, C. J., Title, A. M., van Ballegooijen, A. A., Hagenaar, H. J., \& Shine, R. A. 1997, ApJ, 487, 424

Shine, R. A., Frank, Z. A., Tarbell, T. D., \& Simon, G. W. 2001, in American Geophysical Union, Fall Meeting 2001, A702

Shine, R. A., Simon, G. W., \& Hurlburt, N. E. 2000, Sol. Phys., 193, 313

Simon, G. W., \& Leighton, R. B. 1964, ApJ, 140, 1120

Simon, G. W., Title, A. M., Topka, K. P., et al. 1988, ApJ, 327, 964

Simon, G. W., Title, A. M., \& Weiss, N. O. 2001, ApJ, 561, 427 (STW01)

Steiner, O., Pneuman, G. W., \& Stenflo, J. O. 1986, A\&A, 170, 126

Strous, L. H. 1995, in Helioseismology, ed. J. Hoeksema, V. Domingo, B. Fleck, \& B. Battrick, Proc. of 4th Soho Workshop (ESA SP-376), 213P

Stuart, F. E., \& Rush, J. H. 1954, ApJ, 120, 245

Title, A. M., \& Berger, T. E. 1996, ApJ, 463, 797

Wang, H., Tang, F., Zirin, H., \& Wang, J. 1996, Sol. Phys., 165, 223

Wang, J., Wang, J., Tang, F., Lee, J. W., \& Zirin, H. 1995, Sol. Phys., 160,277

Zhang, J., Lin, G., Wang, J., Wang, H., \& Zirin, H. 1998, A\&A, 338, 322 